\title{
Water as a Quantum Computing Device (POSTER)
}

\author{
Edward G. Belaga ${ }^{1}$, Daniel Grucker ${ }^{2}$, Tarek Khalil ${ }^{3}$, Jean Richert ${ }^{4}$, \\ and Kees van Schenk Brill ${ }^{1,2}$ \\ ${ }^{1}$ IRMA, UMR 7501 CNRS/UDS, 67085 Strasbourg Cedex, France \\ ${ }^{2}$ LINC, UMR 7191 CNRS/UDS, 67084 Strasbourg Cedex, France \\ ${ }^{3}$ Dpt. of Physics, Fac. of Sciences (V), Lebanese University, Nabatieh, Lebanon \\ ${ }^{4}$ Laboratoire de Physique Théorique, UMR 7085 CNRS/ULP, \\ 67084 Strasbourg Cedex, France \\ edward.belaga@math.u-strasbg.fr, schenkbr@math.u-strasbg.fr, \\ grucker@linc.u-strasbg.fr \\ khaliltarek@hotmail.com, richert@fresnel.u-strasbg.fr
}

\begin{abstract}
We propose a new approach in Nuclear Magnetic Resonance (NMR) technology for quantum computing. Two basic elements for quantum computation are qubits and interaction. Traditionnally in NMR, qubits are obtained by considering the spin states of certain atoms of a specific molecule. The interaction that is necessary to create qubit gates such as Control-NOT is then made possible by the spin-spin coupling that exists in such a molecule. One of the drawbacks of this method is the low scalability. More qubits usually means finding an entire different molecule to label the qubits.

We take a different view on the NMR approach. We use a water tube as quantum computer where qubits are defined by the spins which have the same frequency resonance in a small interval defined by the NMR linewidth. Two fundamental roadblocks have to be crossed before this method can even be considered as a possible quantum computation technique: single qubits need to be identified and adressed and an interaction between these qubits has to exist to create two-qubit gates.

We settle the first of these problems by using a magnetic field gradient applied in the main magnetic field direction. The application of a magnetic field gradient during the RF pulse induces a rotation only of those spins whose resonant frequency is equal to the bandwidth of the RF pulse, therefore a qubit can be defined by its resonant frequency and manipulated by selective RF pulses. The main advantage of creating qubits in this way is scalability. As qubits are no longer atoms of a specific molecule but segments of our water tube, increasing the number of qubits would hypothetically just mean increasing the number of segments by applying a stronger magnetic field gradient. Another potential advantage can be obtained during the initialisation phase of the qubits. The second roadblock, the problem of creating interaction between qubits, is work in progress. As for now we are investigating the use of the dipoledipole interaction between the spins to generate a coupling between the spins in order to create entanglements.
\end{abstract}

